\title{
A novel mechanism for C1GALT1 in the regulation of gastric cancer progression
}

\author{
Xiaoxia Dong ${ }^{1,2 \dagger}$, Chunli Chen ${ }^{1 \dagger}$, Xinzhou Deng ${ }^{1}$, Yongyu Liu' ${ }^{1}$, Qiwen Duan ${ }^{1}$, Zhen Peng ${ }^{1}$, Zhiguo Luo $^{1 *}$ and
} Li Shen ${ }^{1,2^{*}}$ (D)

\begin{abstract}
Background: Gastric cancer (GC) is a highly aggressive and lethal disease around the world. High expression of core $1 \beta$ 1, 3-galactosyltransferase 1 (C1GALT1), the primary enzyme responsible for protein O-glycosylation, plays a critical role in gastric carcinogenesis. However, proteins that can be O-glycosylated by C1GALT1 in GC have not been completely elucidated. Also, the mechanism leading to its upregulation in GC is currently unknown.

Results: Using public databases and our patient samples, we confirmed that C1GALT1 expression was upregulated at both the mRNA and protein levels in GC tissues. Elevated expression of C1GALT1 protein was closely associated with advanced TNM stage, lymph node metastasis, tumor recurrence, and poor overall survival. With gain- and lossof-function approaches, we demonstrated that C1GALT1 promoted GC cell proliferation, migration, and invasion. By employing lectin pull-down assay and mass spectrometry, integrin a5 was identified as a new downstream target of C1GALT1 in GC. C1GALT1 was able to modify O-linked glycosylation on integrin a5 and thereby modulate the activation of the PI3K/AKT pathway. Functional experiments indicated that integrin a5 inhibition could reverse C1GALT1mediated tumor growth and metastasis both in vitro and in vivo. Moreover, transcription factor SP1 was found to bind to the C1GALT1 promoter region and activated its expression. Further investigation proved that miR-152 negatively regulated C1GALT1 expression by directly binding to its $3^{\prime}$-UTR.
\end{abstract}

Conclusions: Our findings uncover a novel mechanism for C1GALT1 in the regulation of GC progression. Thus, C1GALT1 may serve as a promising target for the diagnosis and treatment of GC.

Keywords: Gastric cancer, Malignant progression, Glycosyltransferase, C1GALT1

\section{Background}

Gastric cancer (GC) is one of the most frequently occurring malignancies in China and worldwide [1,2]. Despite improvements in diagnostic and therapeutic strategies, the prognosis of GC patients remains very poor [3]. The high mortality among GC patients is partially due to its high invasive and metastatic ability [4]. Hence, further

\footnotetext{
*Correspondence: zhiguo_luo@163.com; 20101061@hbmu.edu.cn ${ }^{\dagger} X i a o x i a$ Dong and Chunli Chen contributed equally to this article 1 Department of Clinical Oncology, Taihe Hospital, Hubei University of Medicine, Shiyan 442000, Hubei, China

Full list of author information is available at the end of the article
}

insight into the mechanisms underlying GC occurrence and progression is becoming increasingly important.

Glycosylation is a ubiquitous and important posttranslational modification. Most of the proteins can be glycosylated with $N$-linked (attached to Asn) or $O$-linked (attached to Ser or Thr) glycans. As a hallmark of cancers, abnormal glycosylation leads to the formation of tumor-associated glycans or glycoproteins [5, 6]. Glycosylation is mainly controlled by the actions of glycosyltransferases, and altered expression of glycosyltransferases results in the production of specific glycans on proteins [7]. Until now, about 300 glycosyltransferases have been identified in the Carbohydrate Active enzyme database. Glycosyltransferases and alterations in protein original author(s) and the source, provide a link to the Creative Commons licence, and indicate if changes were made. The images or other third party material in this article are included in the article's Creative Commons licence, unless indicated otherwise in a credit line to the material. If material is not included in the article's Creative Commons licence and your intended use is not permitted by statutory regulation or exceeds the permitted use, you will need to obtain permission directly from the copyright holder. To view a copy of this licence, visit http://creativecommons.org/licenses/by/4.0/. The Creative Commons Public Domain Dedication waiver (http://creativecommons.org/publicdomain/zero/1.0/) applies to the data made available in this article, unless otherwise stated in a credit line to the data. 
glycosylation have crucial functions in various pathological processes [8-10]. Core $1 \beta 1$, 3-galactosyltransferase 1 (C1GALT1) is the only enzyme that is responsible for the biosynthesis of core $1 O$-glycans (Gal $\beta 1$-3GalNAc $\alpha 1-S e r /$ Thr). C1GALT1 controls the crucial step of GalNAc-type $\mathrm{O}$-glycosylation and is a key contributor to human oncogenesis [11-14]. A recent study reported that C1GALT1 expression was elevated in GC and correlated with gastric carcinogenesis [15]. However, proteins that can be $O$-glycosylated by C1GALT1 in GC have not been completely elucidated. Also, the factors that contribute to its upregulation in GC are currently unknown.

The present study aimed to discover the regulatory mechanisms of C1GALT1 in GC progression. Our results may help to develop novel prognostic biomarkers and potential therapeutic targets for GC.

\section{Results}

\section{C1GALT1 is overexpressed in GC and predicts poor prognosis}

To get an overall profile of C1GALT1 expression in different cancers, we analyzed the expression levels of C1GALT1 mRNA in 33 types of cancers using RNAsequencing data derived from the Cancer Genome Atlas (TCGA) and Genotype-Tissue Expression (GTEx) databases. It showed that C1GALT1 mRNA was overexpressed in a variety of tumors, including GC (Fig. 1a). Then qRT-PCR was performed using fresh-frozen tissues to validate the expression of C1GALT1 mRNA in GC. We found that C1GALT1 mRNA in GC tissues displayed higher levels than in matched adjacent non-tumor tissues (Fig. 1b). The overexpression of C1GALT1 in GC tissues was confirmed at the protein level by Western blot and IHC (Fig. 1c, d). C1GALT1 expression was significantly associated with TNM stage, lymph node metastasis, and tumor recurrence (Table 1). Survival analysis suggested that high C1GALT1 expression was remarkably correlated with reduced overall survival of GC patients (Fig. 1e). Consistently, an online Kaplan-Meier plotter indicated that GC patients with high expression of C1GALT1 had shorter overall survival (Fig. 1f). Univariate analysis identified four factors associated with prognosis: TNM stage, lymph node metastasis, tumor recurrence, and C1GALT1 expression. Multivariate analysis revealed that C1GALT1 was an independent unfavorable prognostic factor (Table 2). These results highlight the importance of C1GALT1 for GC tumorigenesis and prognosis.

\section{C1GALT1 promotes the proliferation, migration, and invasion of GC cells}

To elucidate the functional role of C1GALT1 in GC, the expression profile data of $38 \mathrm{GC}$ cell lines from the
Cancer Cell Line Encyclopedia (CCLE) website were downloaded. The results showed that C1GALT1 mRNA was constitutively expressed in all GC cell lines, but at very different levels (Fig. 2a). Meanwhile, qRT-PCR and Western blot analysis of C1GALT1 expression in one normal gastric cell line and six GC cell lines revealed that C1GALT1 was more frequently overexpressed in GC cell lines than that in normal cell lines (Fig. 2b, c). The relatively higher levels of C1GALT1 were found in MGC803 and BGC-823 cells, whereas SGC-7901 and HGC-27 cells expressed lower levels. Thus, MGC-803 and BGC823 cells were utilized for loss-of-function experiments, whereas SGC-7901 and HGC27 cells were employed for gain-of-function experiments (Fig. 2d, e). CCK-8, Transwell migration, and Matrigel invasion assays showed that overexpression or knockdown of C1GALT1 elevated or suppressed the proliferation, migration, and invasion of GC cells, respectively (Fig. $2 \mathrm{f}-\mathrm{h}$ ). These findings further confirm that C1GALT1 contributes to GC growth and metastasis, which is consistent with a previous report using other GC cell lines [15].

\section{Integrin a5 is a target glycoprotein of C1GALT1 in GC}

To identify the downstream effectors of C1GALT1 in GC, the membrane extracts from MGC-803 and BGC823 cells were prepared and $O$-glycosylated proteins were enriched using lectin PNA. After proteomic analysis and data filtration, 25 and 19 potential PNA-binding proteins were identified in MGC-803 and BGC-823 cells, respectively, 13 of which were common to both cell lines (Fig. 3a, Additional file 1: Table S1). Notably, integrin $\alpha 5$ could be detected in both cell lines. Integrin $\alpha 5$ has been revealed to be involved in cancer development and progression [16-18]. Bioinformatics analysis indicated that integrin $\alpha 5$ was closely related to GC, but its relationship with C1GALT1 has not yet been reported $[19,20]$. Therefore, integrin $\alpha 5$ was selected as a candidate to study. Next, we examined the glycosylation state of integrin $\alpha 5$. We found that overexpression or knockdown of C1GALT1 increased or decreased the binding of PNA to cell-surface and integrin $\alpha 5$ (Fig. 3b, c). Meanwhile, C1GALT1 had no obvious effect on the protein expression of integrin $\alpha 5$. Thus, the interaction between C1GALT1 and integrin $\alpha 5$ is bridged by $O$-glycosylation.

Integrin $\alpha 5$ is an upstream regulator of the PI3K/ AKT pathway [19]. We then investigated whether C1GALT1 was associated with the regulation of integrin $\alpha 5 / \mathrm{PI} 3 \mathrm{~K} / \mathrm{AKT}$ axis in GC cells. Western blot analysis showed that the C1GALT1 knockdown was able to inhibit the activation of the PI3K/AKT pathway (Fig. 3d). Moreover, PI3K/AKT pathway could be activated by C1GALT1 overexpression, and this tendency was blocked by integrin $\alpha 5$ inhibition. Besides, 


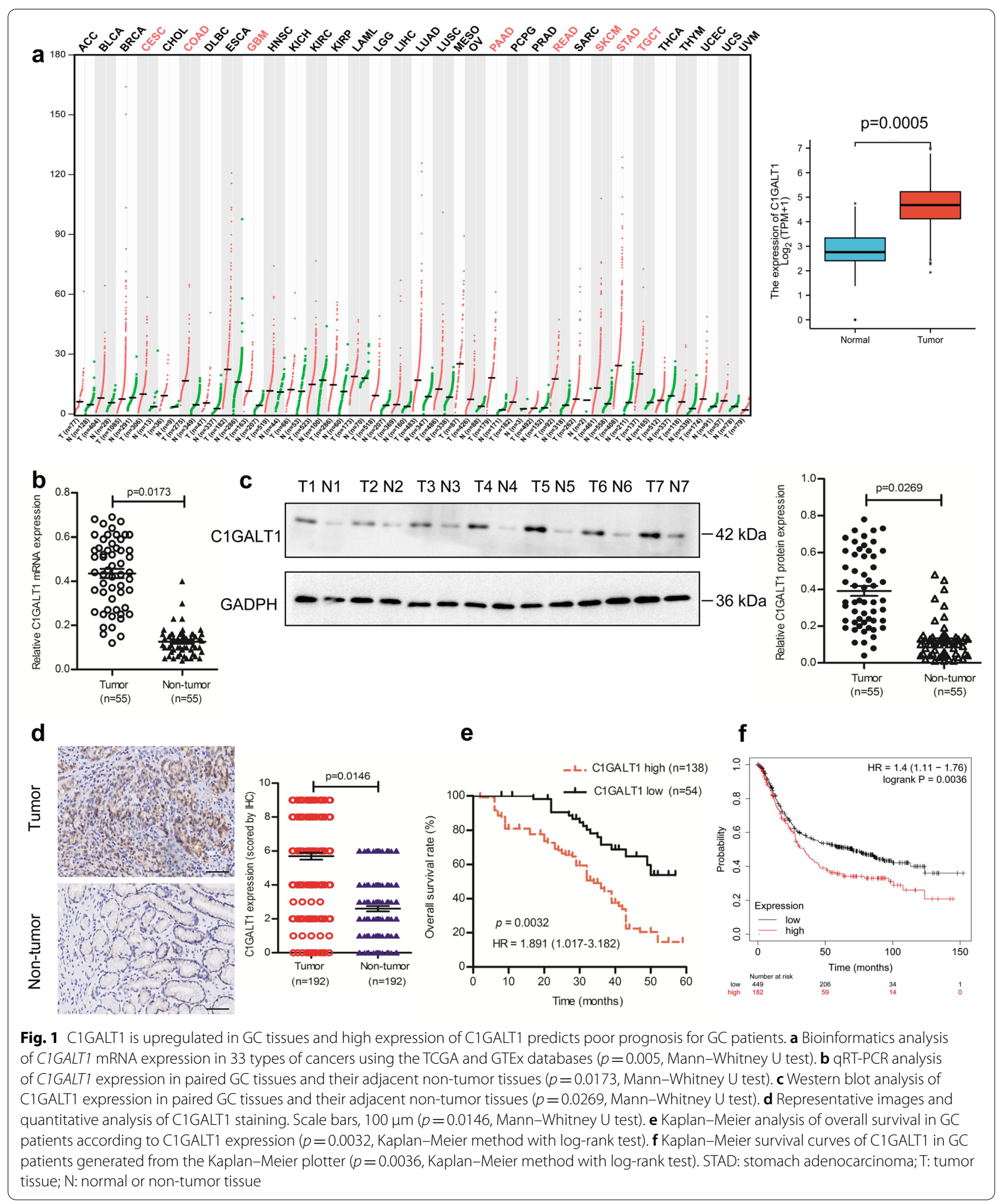


Table 1 Association between C1GALT1 expression and clinicopathological parameters in GC

\begin{tabular}{|c|c|c|c|c|}
\hline \multirow[t]{2}{*}{ Variables } & \multirow{2}{*}{$\begin{array}{l}\text { Number } \\
\text { of cases }\end{array}$} & \multicolumn{2}{|l|}{ C1GALT1 } & \multirow[t]{2}{*}{ P-value } \\
\hline & & Low (54) & High (138) & \\
\hline \multicolumn{5}{|l|}{ Age (years) } \\
\hline$<60$ & 60 & 15 & 45 & 0.072 \\
\hline$\geq 60$ & 132 & 39 & 93 & \\
\hline \multicolumn{5}{|l|}{ Gender } \\
\hline Male & 116 & 30 & 86 & 0.351 \\
\hline Female & 76 & 24 & 52 & \\
\hline \multicolumn{5}{|l|}{ Tumor size (cm) } \\
\hline$<5$ & 68 & 18 & 50 & 0.533 \\
\hline$\geq 5$ & 124 & 36 & 88 & \\
\hline \multicolumn{5}{|l|}{ Differentiation } \\
\hline Well, moderately & 51 & 10 & 41 & 0.196 \\
\hline Poorly & 141 & 44 & 97 & \\
\hline \multicolumn{5}{|l|}{ TNM stage } \\
\hline $1+\|$ & 87 & 37 & 50 & $0.039^{*}$ \\
\hline$I I I+I V$ & 105 & 17 & 88 & \\
\hline \multicolumn{5}{|l|}{ Grade } \\
\hline $1-2$ & 82 & 23 & 59 & 0.062 \\
\hline $3-4$ & 110 & 31 & 79 & \\
\hline \multicolumn{5}{|l|}{ Recurrence } \\
\hline Absent & 88 & 32 & 46 & $0.011^{*}$ \\
\hline Present & 104 & 22 & 92 & \\
\hline \multicolumn{5}{|c|}{ Lymph node metastasis } \\
\hline Negative & 93 & 45 & 48 & $0.005^{*}$ \\
\hline Positive & 99 & 9 & 90 & \\
\hline
\end{tabular}

correlation studies in GC tissues demonstrated that C1GALT1 was weakly correlated with integrin $\alpha 5$ but strongly correlated with p-PI3K and p-AKT (Fig. 3e, f). These results suggest that C1GALT1 activates PI3K/ AKT pathway in GC, which may be dependent on $O$-glycosylation of integrin $\alpha 5$.

\section{Integrin a5 is a key mediator in C1GALT1-induced GC growth and metastasis}

To explore the functional relevance of integrin $\alpha 5$ in C1GALT1-mediated GC progression, CCK-8, Transwell migration, and Matrigel invasion assays were performed. We found that inhibition of integrin $\alpha 5$ attenuated the potentiation effects of C1GALT1 overexpression on GC cell proliferation, migration, and invasion (Fig. $4 \mathrm{a}-\mathrm{c}$ ). To validate our in vitro results, we established a subcutaneous tumor model and a peritoneal metastatic xenograft model in nude mice, respectively. The results showed that the promotive effects of C1GALT1 overexpression on tumor growth were weakened by integrin $\alpha 5$ inhibition (Fig. 4d, e). Furthermore, C1GALT1 overexpression led to a significant increase in the number of visible peritoneal nodules. In contrast, integrin $\alpha 5$ inhibition reduced the increased visible peritoneal nodules induced by C1GALT1 (Fig. 4f). Overall, our data support integrin $\alpha 5$ as a functionally important target protein of C1GALT1 in GC.

Table 2 Univariate and multivariate analysis for GC patients using the Cox regression model

\begin{tabular}{|c|c|c|c|c|}
\hline \multirow[t]{2}{*}{ Variables } & \multicolumn{2}{|l|}{ Univariate analysis } & \multicolumn{2}{|l|}{ Multivariate analysis } \\
\hline & $\mathrm{HR}(95 \% \mathrm{CI})$ & P-value & $\mathrm{HR}(95 \% \mathrm{Cl})$ & P-value \\
\hline $\begin{array}{l}\text { Gender } \\
\text { Male vs. Female }\end{array}$ & $1.068(0.729-1.602)$ & 0.831 & $1.302(0.746-2.267)$ & 0.324 \\
\hline $\begin{array}{l}\text { Age (years) } \\
<60 \text { vs. } \geq 60\end{array}$ & $0.725(0.446-1.189)$ & 0.603 & $0.876(0.535-1.434)$ & 0.775 \\
\hline $\begin{array}{l}\text { Tumor size }(\mathrm{cm}) \\
<5 \text { vs. } \geq 5\end{array}$ & 1.039 (0.534-1.713) & 0.292 & $0.914(0.455-1.842)$ & 0.802 \\
\hline $\begin{array}{l}\text { Differentiation } \\
\text { Well and moderately vs. Poorly }\end{array}$ & 1.577 (1.048-1.959) & 0.304 & 1.539 (0.799-2.966) & 0.181 \\
\hline $\begin{array}{l}\text { TNM stage } \\
\text { I+ II vs. III+ IV }\end{array}$ & $2.533(1.529-4.265)$ & $0.009^{*}$ & $2.871(1.506-5.393)$ & 0.063 \\
\hline $\begin{array}{l}\text { Grade } \\
1-2 \text { vs. 3-4 }\end{array}$ & $1.352(0.887-2.008)$ & 0.158 & $1.438(0.779-2.702)$ & 0.261 \\
\hline $\begin{array}{l}\text { Recurrence } \\
\text { Absent vs. Present }\end{array}$ & $2.728(1.695-4.013)$ & $0.025^{*}$ & $2.305(0.922-5.771)$ & 0.052 \\
\hline $\begin{array}{l}\text { Lymph node metastasis } \\
\text { Negative vs. Positive }\end{array}$ & $2.076(1.243-3.022)$ & $0.027^{*}$ & $1.356(0.709-2.174)$ & $0.033^{*}$ \\
\hline $\begin{array}{l}\text { C1GALT1 expression } \\
\text { Low vs. High }\end{array}$ & 1.655 (1.102-2.474) & $0.001^{*}$ & $1.773(1.094-2.565)$ & $0.004^{*}$ \\
\hline
\end{tabular}




\section{(See figure on next page.)}

Fig. 2 C1GALT1 promotes GC cell proliferation, migration, and invasion. a Bioinformatics analysis of C1GALT1 mRNA expression in 38 GC cell lines using the CCLE database. $\mathbf{b}$ qRT-PCR analysis of C1GALT1 expression in one normal gastric cell line and six GC cell lines. $\mathbf{c}$ Western blot analysis of C1GALT1 expression in different cell lines. d C1GALT1 protein expression was detected by Western blot after transfection. e C1GALT1 mRNA expression was analyzed by qRT-PCR after transfection. $\mathbf{f C C K}-8$ assay was used for proliferation analysis. $\mathbf{g}$ Transwell chambers without Matrigel were used for migration analysis. $\mathbf{h}$ Matrigel-coated Transwell chambers were used for invasion analysis. shNC: cells transfected with negative control lentivirus; shRNAs: cells transfected with C1GALT1 shRNA lentivirus; Mock: cells transfected with empty plasmid; OV: cells transfected with C1GALT1 plasmid. ${ }^{*} p<0.05,{ }^{* *} p<0.01$ compared with the shNC or Mock group (Student's t-test or one-way ANOVA)

\section{SP1 transcriptionally upregulates C1GALT1 expression in GC}

Transcription factors are essential for modulating gene expression. The 9 top-ranked transcription factors predicted by the GeneCards platform were ATF-2, c-Jun, GATA-1, HNF-1 A, IKZF2, NFE2L1, MAZR, PPAR- $\alpha$, and SP1. We performed a correlation analysis between these transcription factors and C1GALT1 expression in GC using the TCGA and GTEx datasets. A strong association was observed between C1GALT1 and SP1 but not other transcription factors (Fig. 5a, Additional file 1: Figure S1). IHC analysis of GC tissues showed that SP1 expression was positively correlated with C1GALT1 expression (Fig. 5b). The expression of SP1 in different GC cells exhibited a consistent tendency with that of C1GALT1 (Fig. 5c). Furthermore, C1GALT1 expression was decreased after SP1 knockdown but increased after SP1 overexpression (Fig. 5d, Additional file 1: Figure S2). Considering a specific role for SP1 in the control of C1GALT1 transcription, we next analyzed the promoter region of C1GALT1 via the JASPAR database. Through bioinformatics analysis, two potential SP1 binding sites $(-676 /-666 ;-67 /-57)$ were identified (Fig. 5e). ChIP enrichment analysis indicated that SP1 could bind the C1GALT1 promoter region (Fig. 5f). The dual-luciferase reporter assays revealed that $C 1 G A L T 1$ promoter activity was enhanced or inhibited when SP1 was overexpressed or knocked down, respectively (Fig. 5g). We also found that mutation of either binding site reduced promoter activity, whereas mutation of both binding sites resulted in a complete loss of activity (Fig. 5h). We further discovered that SP1 knockdown inhibited cell proliferation, migration, and invasion. However, these inhibitory effects were reversed by C1GALT1 overexpression (Fig. 5i-k). Collectively, these results imply that SP1 is required for regulating C1GALT1 expression in GC.

\section{Decreased miR-152 contributes to C1GALT1 overexpression in GC}

MiRNAs are generally considered to post-transcriptionally and negatively regulate gene expression. To explore whether the expression of C1GALT1 was regulated by specific miRNAs, three bioinformatics software (TargetScan, miRanda, and miRDB) were utilized to predict the
miRNAs that can bind to 3'-UTR of C1GALT1 mRNA. We found that C1GALT1 was a predicted target of miR148b and miR-152 (Fig. 6a). Although qRT-PCR analysis revealed that these two miRNAs were down-regulated in GC tissues, only miR-152 was significantly negatively correlated with C1GALT1 expression (Fig. 6b, Additional file 1: Figure S3). C1GALT1 expression was suppressed by overexpression of miR-152 and increased by knockdown of miR-152 (Fig. 6c, d). Phenotypic experiments showed that miR-152 overexpression led to a prominent reduction in cell proliferation, migration, and invasion. Meanwhile, these effects could be reversed by the restoration of C1GALT1 (Fig. 6e-g). Sequence analysis demonstrated that the $3^{\prime}$-UTR of C1GALT1 mRNA had complementary binding sites with miR-152 (Fig. 6h). Moreover, miR-152 overexpression repressed the luciferase activity of the wild-type but not the mutant 3'-UTR of C1GALT1 (Fig. 6i). Thus, miR-152 functions as a negative regulator of C1GALT1 in GC.

\section{Discussion}

The present study investigated the clinical significance and biological function of C1GALT1 in GC. The downstream effectors (O-glycosylated proteins) and upstream regulators (transcription factors and miRNAs) of C1GALT1 in GC were also explored. C1GALT1 was found to be overexpressed in GC and was a marker of poor prognosis. C1GALT1 played a central role in the malignant progression of $\mathrm{GC}$ via modifying integrin $\alpha 5$ $O$-glycosylation and activating the PI3K/AKT pathway. Moreover, C1GALT1 was transcriptionally upregulated by SP1, and decreased miR-152 further contributed to C1GALT1 mRNA stability in GC (Fig. 7). Our findings uncover a novel mechanism for C1GALT1 in promoting GC progression. To our knowledge, this is the first study to explore the detailed mechanism of C1GALT1 upregulation in GC. The regulatory relationship between C1GALT1 and integrin $\alpha 5$ was also firstly reported in GC.

Altered expression of glycosyltransferases is crucial in determining the development, progression, and aggressiveness of GC [21-23]. A previous study analyzed the association between C1GALT1 expression and clinical characteristics of 98 GC patients by IHC [15]. The 


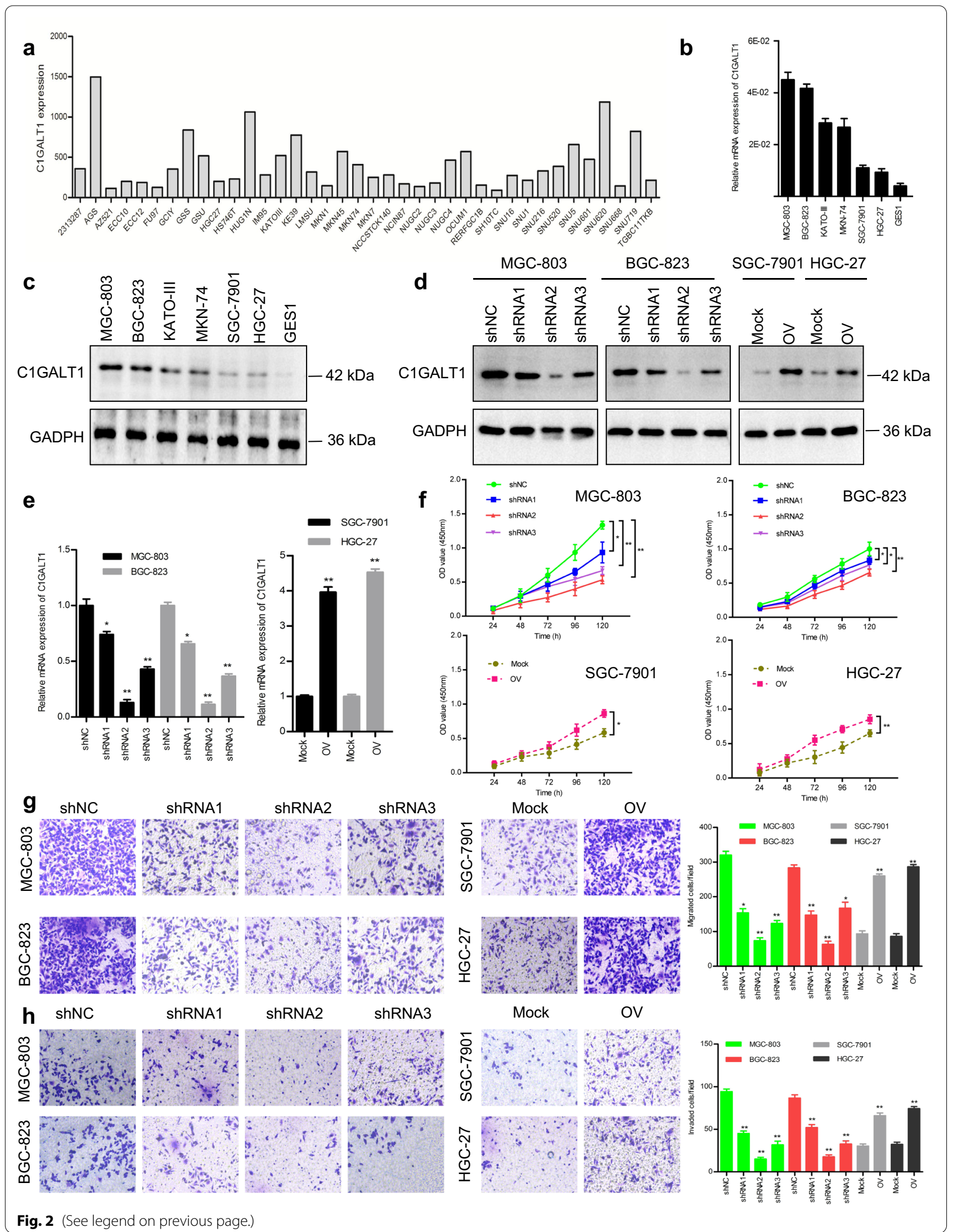


(See figure on next page.)

Fig. 3 C1GALT1 regulates the O-glycosylation of integrin a 5 in GC. a The number of PNA-binding proteins identified by mass spectrometry was displayed using Venny. $\mathbf{b}$ The cell-surface proteins that interacted with PNA were detected using a lectin-based pull-down assay. Membrane proteins were divided into two parts, one for GADPH detection, and the other for PNA-binding measurement. GAPDH was an internal control. c The proteins from whole-cell lysates enriched by PNA were analyzed by immunoblotting with an anti-integrin a 5 antibody. $\mathbf{d}$ The indicated protein expression was evaluated by Western blot after transfection. e Representative images of integrin a5, p-PI3K, and p-AKT staining in GC samples. Scale bars, $100 \mu \mathrm{m}$. $\mathbf{f}$ Correlation analysis (Spearman's rank correlation test). shNC: cells transfected with negative control lentivirus; shRNA: cells transfected with C1GALT1 shRNA2 lentivirus; Mock: cells transfected with empty plasmid; OV: cells transfected with C1GALT1 plasmid; siNC: cells transfected with negative control siRNA; siRNA: cells transfected with integrin a5 siRNA; IB: immunoblot; PD: pull-down

current study analyzed a series of public databases, including TCGA, GTEx, and the Kaplan-Meier plotter, to get an overall expression profile and prognostic value of C1GALT1 in GC. Besides, qRT-PCR, Western blot, and IHC were performed in $192 \mathrm{GC}$ samples to verify the findings obtained from the bioinformatics analysis. Using different vectors, cell types, and animal models, we further provide evidence indicating that C1GALT1 can function as an important contributor to GC growth and metastasis.

The downstream events following C1GALT1 dysregulation are linked to the alteration in protein $O$-glycosylation. For example, knockout of C1GALT1 led to the truncation of $O$-glycosylation on MUC16 in pancreatic adenocarcinoma [24]. O-glycosylation of EGFR was blocked by C1GALT1 knockdown in head and neck cancer [11]. C1GALT1 modified the $O$-glycosylation of MET in hepatocellular carcinoma [25]. These studies also confirmed that altered $O$-glycosylation was closely associated with tumor proliferation, invasion, and metastasis. In this study, we discovered that integrin $\alpha 5$ was a potential substrate for C1GALT1 during GC progression. Integrins are a family of transmembrane glycoprotein receptors, which contain both $N$ - and $O$-linked glycosylation sites [26]. Integrin $\alpha 5 \mathrm{~N}$-glycosylation plays a crucial role in multiple biological processes, including cell adhesion and migration [27, 28]. However, the mechanism of integrin a5 O-glycosylation in GC remains unclear. Using lectin pull-down assay, we found that C1GALT1 overexpression increased, while knockdown decreased, the $O$-glycosylation of integrin $\alpha 5$. Functional experiments indicated that integrin $\alpha 5$ inhibition could reverse C1GALT1-mediated tumor growth and metastasis both in vitro and in vivo. Thus, integrin $\alpha 5$ is a key mediator in C1GALT1-induced GC progression. In addition to integrin $\alpha 5$, our studies also supply many other glycoproteins that still need to be explored.

So far, the underlying mechanism accounting for C1GALT1 upregulation in GC remains not completely understood. SP1 is an essential transcription factor for gene regulation and can drive the expression of many cancer-related genes. It is widely reported that abnormal expression of SP1 contributed to GC tumorigenesis
$[4,29,30]$. In the present study, paralleled expression of C1GALT1 and SP1 in GC was observed. SP1 was a positive regulator of C1GALT1 via binding to its promoter. miR-152 is a type of abnormally expressed miRNAs in many types of malignancies including GC [31-33]. We found that the expression of miR-152 was negatively correlated with that of C1GALT1 in GC. miR-152 served as a negative regulator of C1GALT1 via direct interaction with its 3'-UTR. Despite the large number of target genes of SP1 and miR-152 have been identified, the relationship between these two molecules and C1GALT1 is still unknown. Our study provides the first experimental evidence that SP1 and miR-152 act in concert to promote GC growth and metastasis by regulating C1GALT1.

There are several limitations to our study. For instance, the number of tissue specimens that we have studied is not large, although substantial. We have shown the presence of integrin $\alpha 5$ in GC tissues. Unfortunately, we are unable to confirm the expression of $O$-glycosylated integrin $\alpha 5$ in these samples. In addition, it is currently unclear which sites on integrin $\alpha 5$ are $O$-glycosylated by C1GALT1. Moreover, the mechanism of synergy between SP1 and miR-152 requires further investigation. It would be of interest to investigate whether these findings can be extended to other tumors.

\section{Conclusions}

In summary, we reported that increased C1GALT1 expression was an effective predictor of worse prognosis in GC. C1GALT1 potentiated GC growth and metastasis by targeting integrin $\alpha 5$. C1GALT1 was transcriptionally activated by SP1 and was post-transcriptionally controlled by miR-152. Hence, C1GALT1 may serve as a novel oncogene during GC progression. Our study will provide an important insight into the pathogenesis of GC and supply potential targets for new drug inventions.

\section{Methods}

\section{Patients samples and cell lines}

The Ethics Committee of Hubei University of Medicine (Shiyan, China) approved all research. Archived GC tissue specimens were collected from patients undergoing surgery in Taihe Hospital without radio- or 


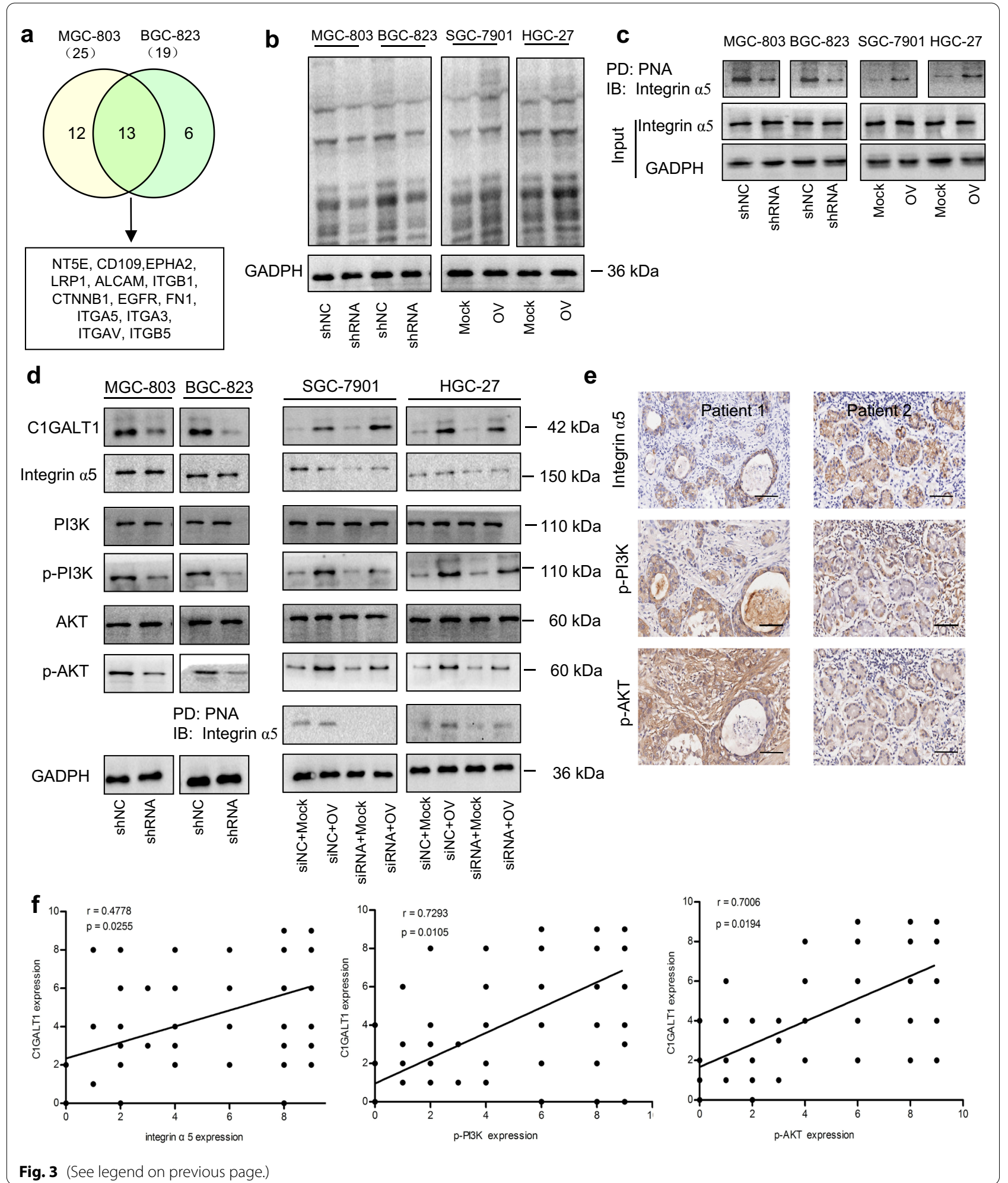

chemotherapy after obtaining written informed consent. The samples were divided into two parts, one was fixed in formalin and another was stored at $-80^{\circ} \mathrm{C}$. GC cell lines
(KATO-III, BGC-823, MGC-803, SGC-7901, MKN-74, HGC-27) and gastric mucosal epithelial cell line (GES-1) were purchased from Procell (Wuhan, China). Cells were 


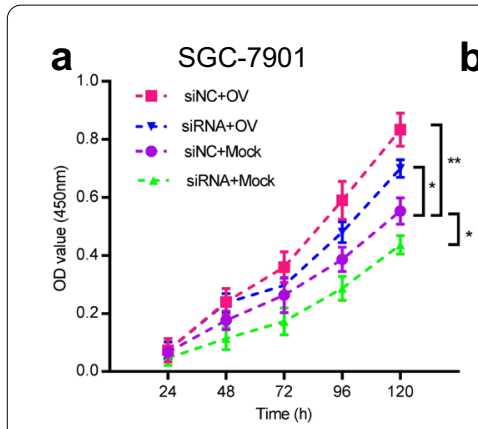

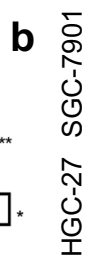
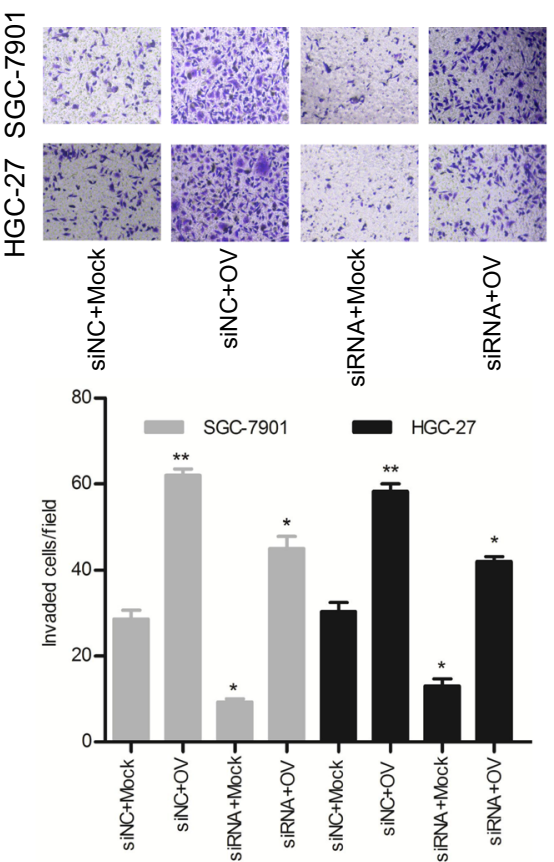

d
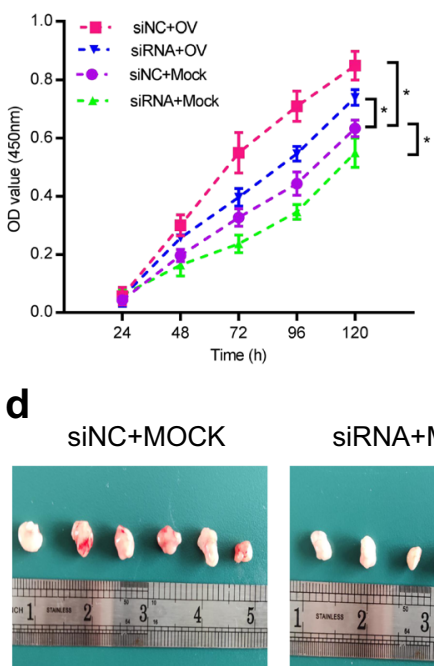

siNC+OV

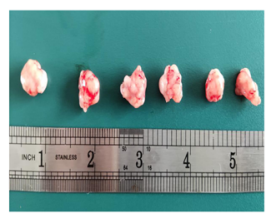

f
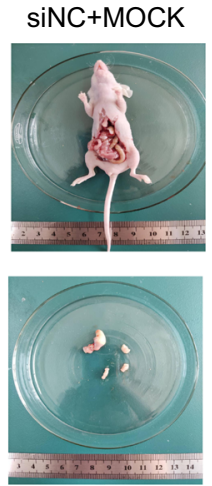

SiRNA+MOCK

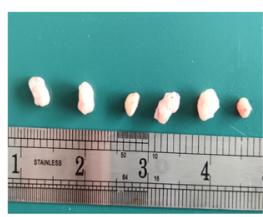

SiRNA+OV
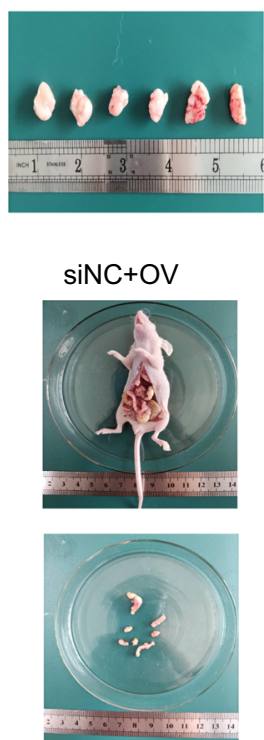

$\mathbf{e}$
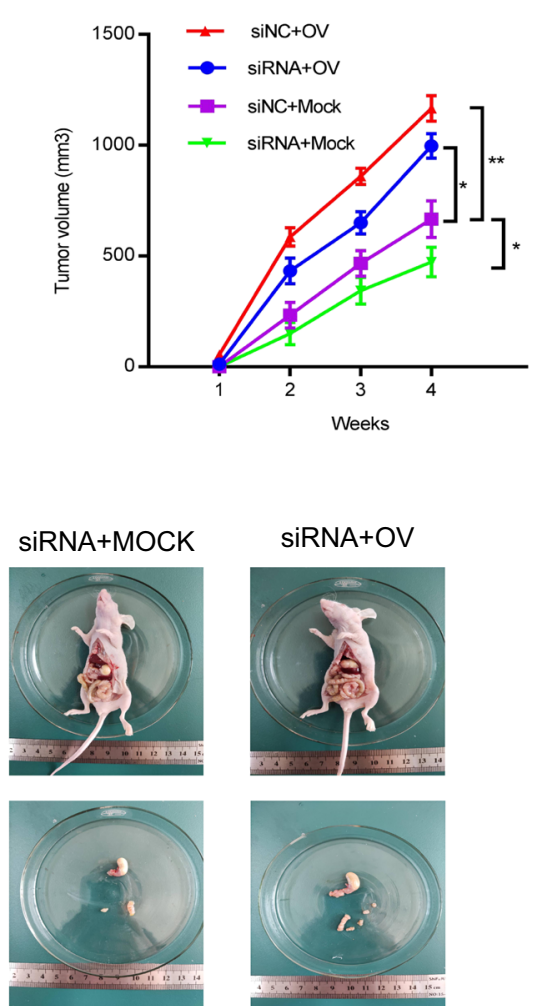

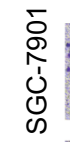
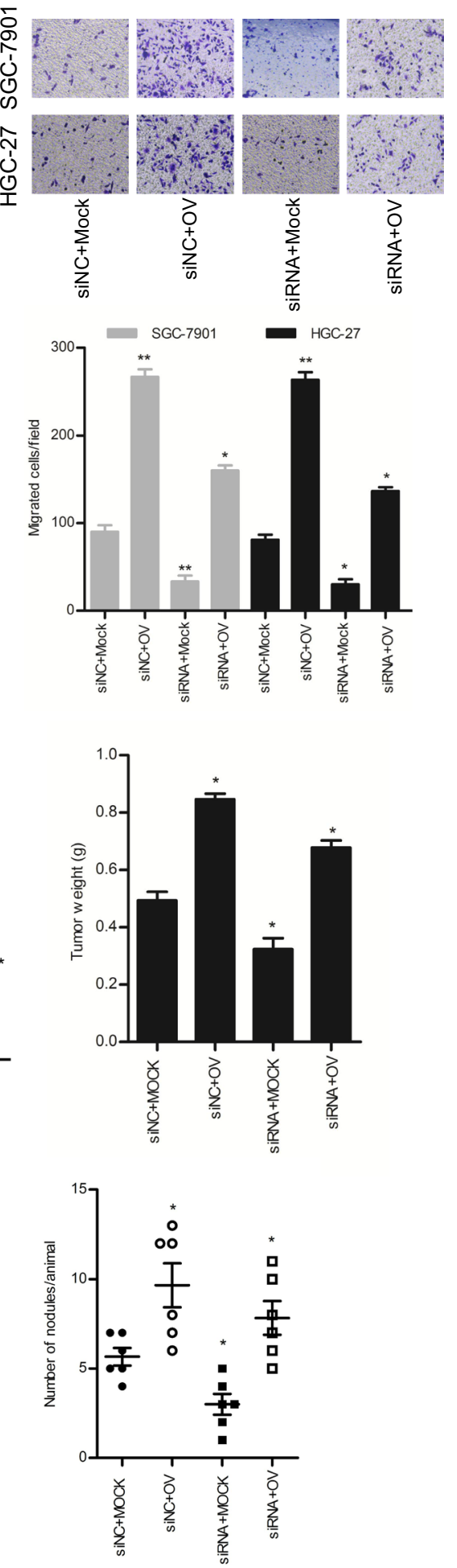

Fig. 4 Effects of C1GALT1 on GC proliferation, migration, and invasion are mediated by integrin a5. a-c Cell proliferation, migration, and invasion were assessed by CCK-8, Transwell migration, and Matrigel invasion assays. $\mathbf{d}$ Photographs of xenograft tumors. e The tumor growth curve and tumor weight in the nude mice. $\mathbf{f}$ Metastatic nodules were photographed and counted. $n=6$ mice per group. Mock: cells transfected with empty plasmid; OV: cells transfected with C1GALT1 plasmid; siNC: cells transfected with negative control siRNA; siRNA: cells transfected with integrin a5 siRNA. ${ }^{*} p<0.05,{ }^{* *} p<0.01$ compared with the siNC+Mock group (Student's t-test or one-way ANOVA) 


\section{(See figure on next page.)}

Fig. 5 C1GALT1 is transcriptionally activated by SP1 in GC. a Scatter diagrams for C1GALT1 expression versus SP1 expression in GC samples based on the TCGA and GTEx databases(Spearman's rank correlation test). b Representative images and quantitative analysis of SP1 expression in GC tissues (Spearman's rank correlation test). Scale bars, $100 \mu \mathrm{m}$. c Western blot analysis of SP1 expression in different GC cells. d The effect of SP1 on C1GALT1 expression was detected by Western blot. e Schematic presentation of SP1 binding sites within the promoter region of C1GALT1. fThe binding of SP1 to the C1GALT1 promoter was analyzed by ChIP-PCR. $\mathbf{g}$, $\mathbf{h}$ Relative luciferase activity was examined by the dual-luciferase reporter assay. i-k Cell proliferation, migration, and invasion were assessed by CCK-8, Transwell migration, and Matrigel invasion assays. siNC: cells transfected with negative control siRNA; si1: cells transfected with SP1 siRNA1; si2, cells transfected with SP1 siRNA2; Ctrl, cells transfected with control plasmid; SP1, cells transfected with SP1 plasmid; OV: cells transfected with C1GALT1 plasmid; WT: Wild-type; MUT: mutant. ${ }^{*} p<0.05$, ${ }^{* *} p<0.01$ compared with the siNC or Ctrl group (Student's t-test or one-way ANOVA)

cultured in RPMI-1640 or Hams F12 medium (Gibco, USA) containing $10 \%$ fetal bovine serum (FBS; Gibco).

\section{Immunohistochemistry (IHC) staining}

IHC was conducted as previously described [34]. The staining degree was calculated as follows: Overall score $=$ intensity score ( 0 , negative; 1 , weak; 2 , moderate; and 3 , strong) $\times$ percentage score $(0,0-5 \% ; 1,6-25 \%$; $2,26-50 \% ; 3,51-75 \% ; 4, \geq 76 \%)$. A final score $\geq 4$ was considered as high expression and $<4$ as low expression. The following antibodies were used: C1GALT1 (ab237734, 1:500, Abcam, USA), integrin $\alpha 5$ (ab150361, 1:100, Abcam), p-PI3K (abs103557, 1:200, Absin, China), p-AKT (abs130889, 1:150, Absin), and SP1 (ab124804, 1:150, Abcam).

\section{Oligonucleotide and plasmid transfection}

C1GALT1 shRNA lentivirus plasmid, C1GALT1 overexpression plasmid, integrin $\alpha 5$ siRNAs, SP1 siRNAs, SP1 overexpression plasmid, miR-152-3p mimics, miR152-3p inhibitor, and their corresponding negative controls were acquired from GenePharma (Suzhou, China). The sequences for shRNAs and siRNAs were listed in Additional file 1: Table S2. Cell transfection was performed using Lipofectamine 3000 (Invitrogen, USA) or siRNA-mate (GenePharma). Transfection efficiency was evaluated by quantitative real-time PCR (qRT-PCR) and Western blot after transfection. Stable cell lines were selected with puromycin (Sigma, USA).

\section{qRT-PCR and western blot}

Total RNA was reverse-transcribed into cDNA using TransScript First-Strand cDNA Synthesis SuperMix (TransGen, China). qRT-PCR reactions were conducted using SYBR PCR Master Mix (ABI, USA). The primers were listed in Additional file 1: Table S3. The relative gene expression was normalized to control using the $2^{-\Delta \Delta \mathrm{Ct}}$ method. Total protein was quantified using the BCA protein assay kit (Biosharp, China). The approach for Western blot was conducted as described previously [34]. The following antibodies were used: C1GALT1 (ab237734, Abcam), integrin a5 (ab52971, Abcam), PI3K
(4255, CST, USA), p-PI3K (4228, CST), AKT (9272, CST), p-AKT (9271, CST), SP1 (ab124804, Abcam), and GADPH (BL006B, Biosharp).

\section{Cell proliferation, migration, and invasion assays}

Cells $\left(2 \times 10^{3} /\right.$ well $)$ were seeded into 96 -well plates and cell viability was examined at 24, 48, 72, and $96 \mathrm{~h}$ using Cell Counting Kit-8 (CCK-8; Dojindo, Japan). The migratory and invasive abilities were measured using 8- $\mu \mathrm{m}$ transwell chambers (24-well insert, Corning, USA) coated with (invasion assay) or without (migration assay) Matrigel (BD Biosciences, USA), respectively. $3 \times 10^{4}$ cells in serum-free medium were seeded in the upper chamber. After $24 \mathrm{~h}$ (migration assay) or $36 \mathrm{~h}$ (invasion assay), cells on the lower side of the chamber were stained and photographed.

\section{Lectin pull-down assay and mass spectrometry analysis}

Membrane protein was extracted using a CelLytic MEM Protein Extraction kit (Sigma) and then incubated overnight with peanut agglutinin (PNA)-coated agarose beads (Vector Labs, USA) at $4{ }^{\circ} \mathrm{C}$. The pulled-down proteins were subjected to $10 \%$ SDS-PAGE. For mass spectrometry analysis, the gels were stained with coomassie brilliant blue. Mass spectrometry was carried out based on the method as already described by us and others [8, 35]. For analysis of the modifications to cell-surface glycoproteins, proteins in the gels were electrophoretically transferred to a PVDF membrane (Millipore, USA). The membrane was probed with biotin-labeled PNA (Vector Labs). Subsequently, bands were visualized using HRPconjugated streptavidin (Vector Labs). To evaluate the glycosylation status of integrin $\alpha 5$, total proteins from whole-cell lysates were prepared. After incubation with PNA-coated beads, the precipitated complexes were separated by SDS-PAGE and immunoblotted with the antibody against integrin $\alpha 5$.

\section{Chromatin immunoprecipitation (ChIP) assay}

ChIP was performed using the EZ-ChIP ${ }^{\mathrm{TM}}$ Kit (Millipore, USA) [36]. DNA fragments were generated by ultrasound, followed by incubation with an anti-SP1 antibody 
a

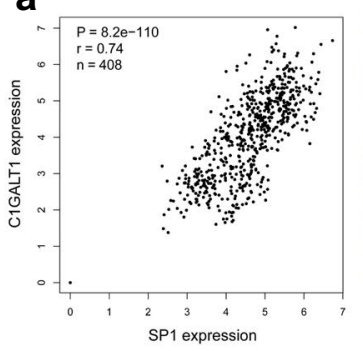

C

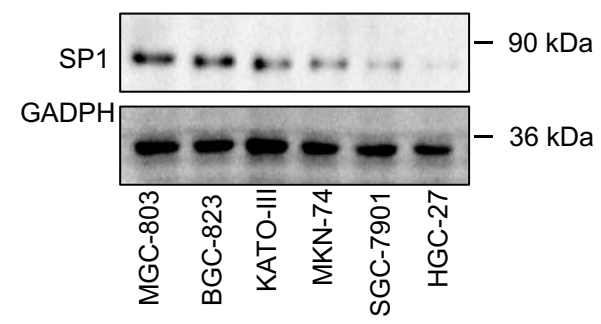

b $\quad$ Tumor

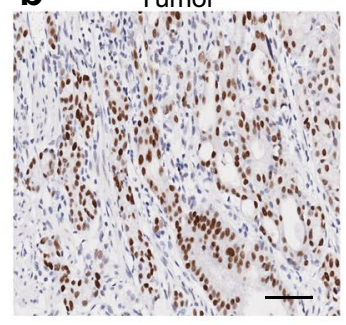

\section{d}

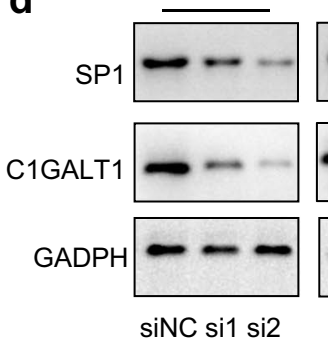

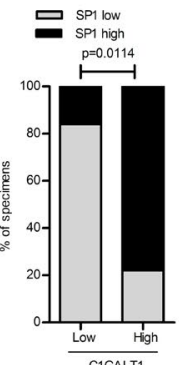

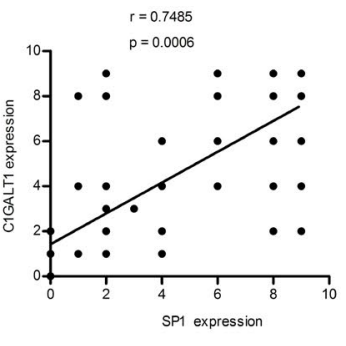

e

SP1 DNA binding motif
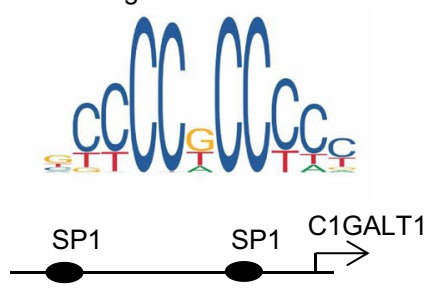

$-676 \mathrm{bp}--666 \mathrm{bp}$ Binding site 1 ССТССТСССC $-67 b p--57 b p$ Binding site 2 TCCCCGCCCA

h
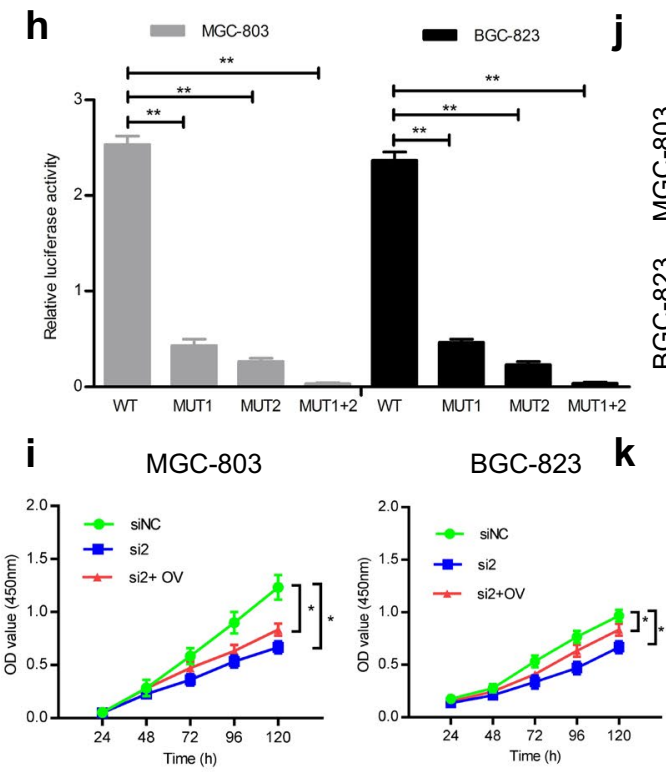

f

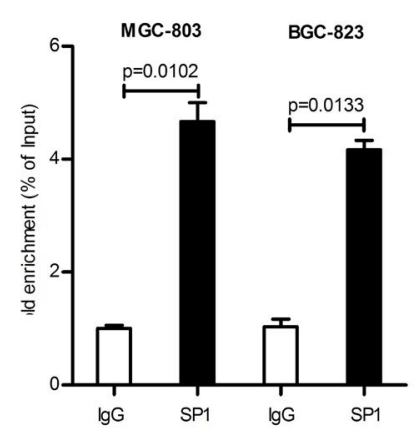

BGC-823 SGC-7901 HGC-27

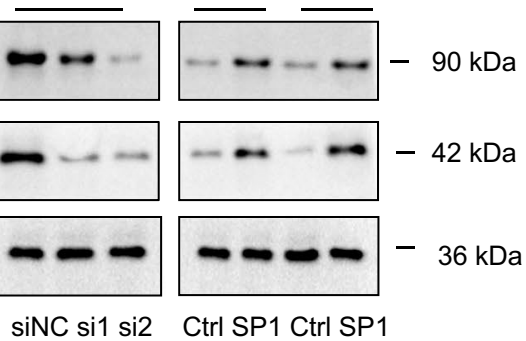

g

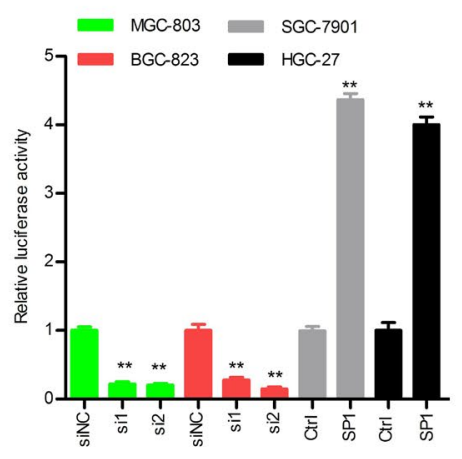

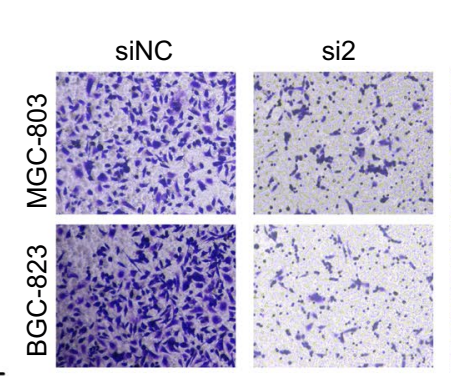

siNC

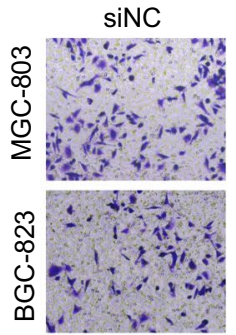

si2
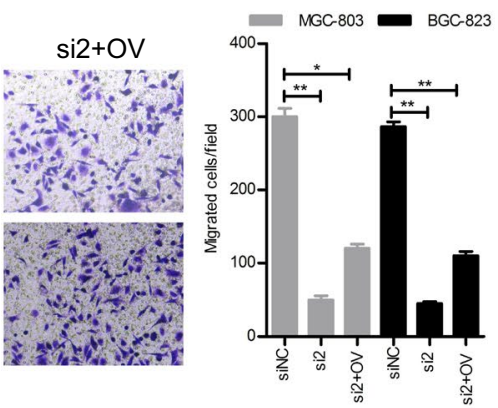
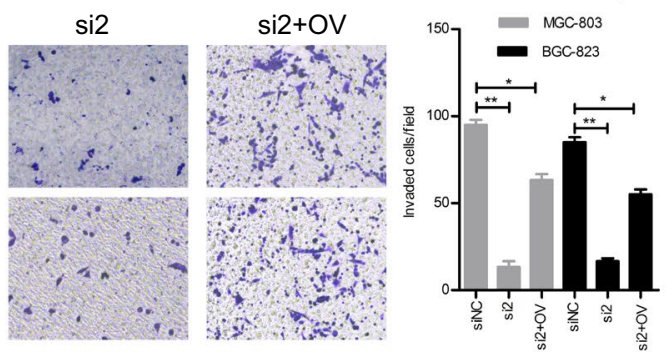

Fig. 5 (See legend on previous page.) 


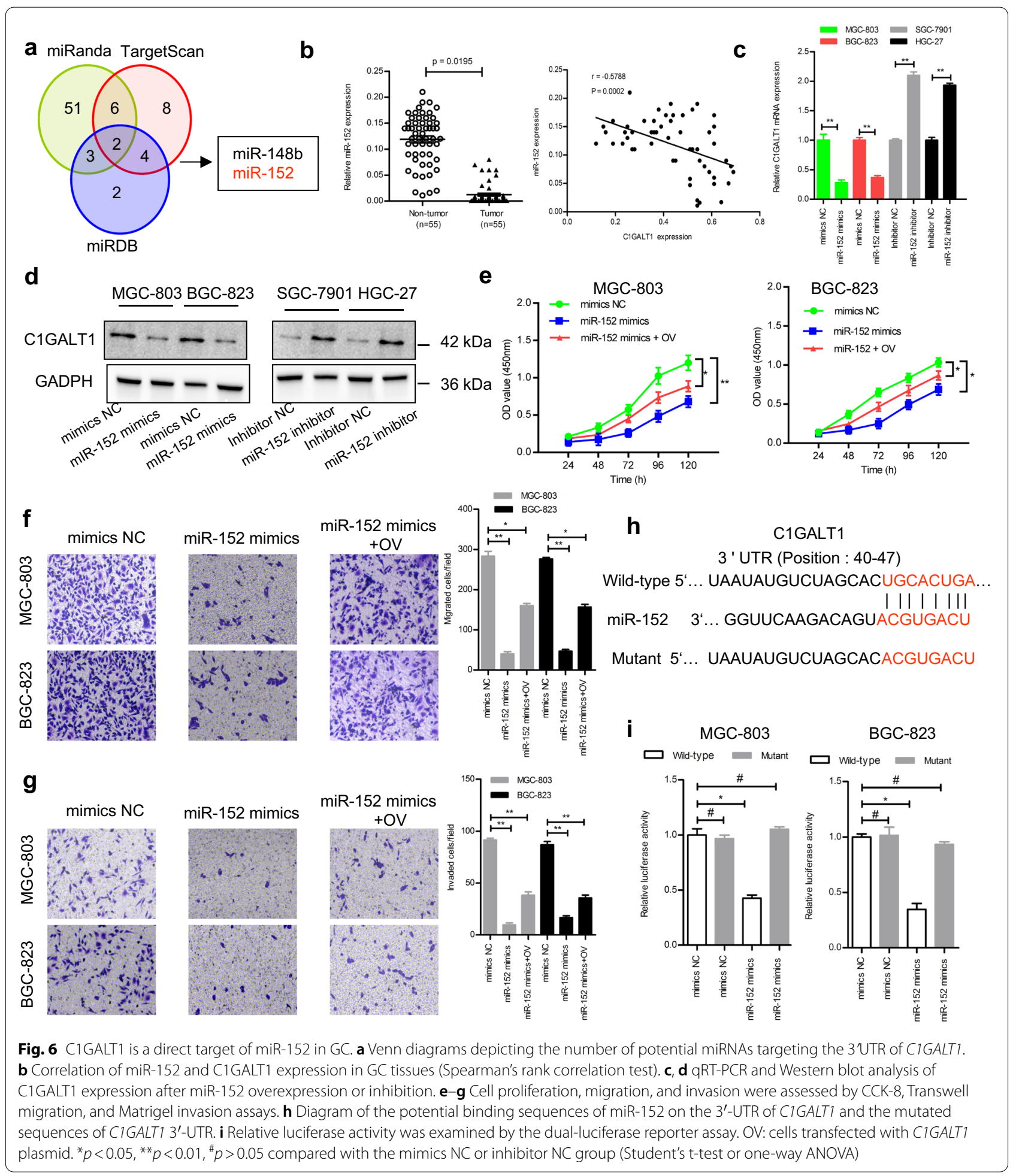

(ab231778, Abcam) or IgG isotype antibody (ab172730, Abcam). DNA recovered from reverse cross-linking was used for qRT-PCR. The primers were listed in Additional file 1: Table S3.

\section{Luciferase reporter assay}

The C1GALT1 wild-type or mutant 3'-UTR was constructed and cloned into a pGL3 luciferase reporter plasmid (Promega, USA). Then the vectors combined with 


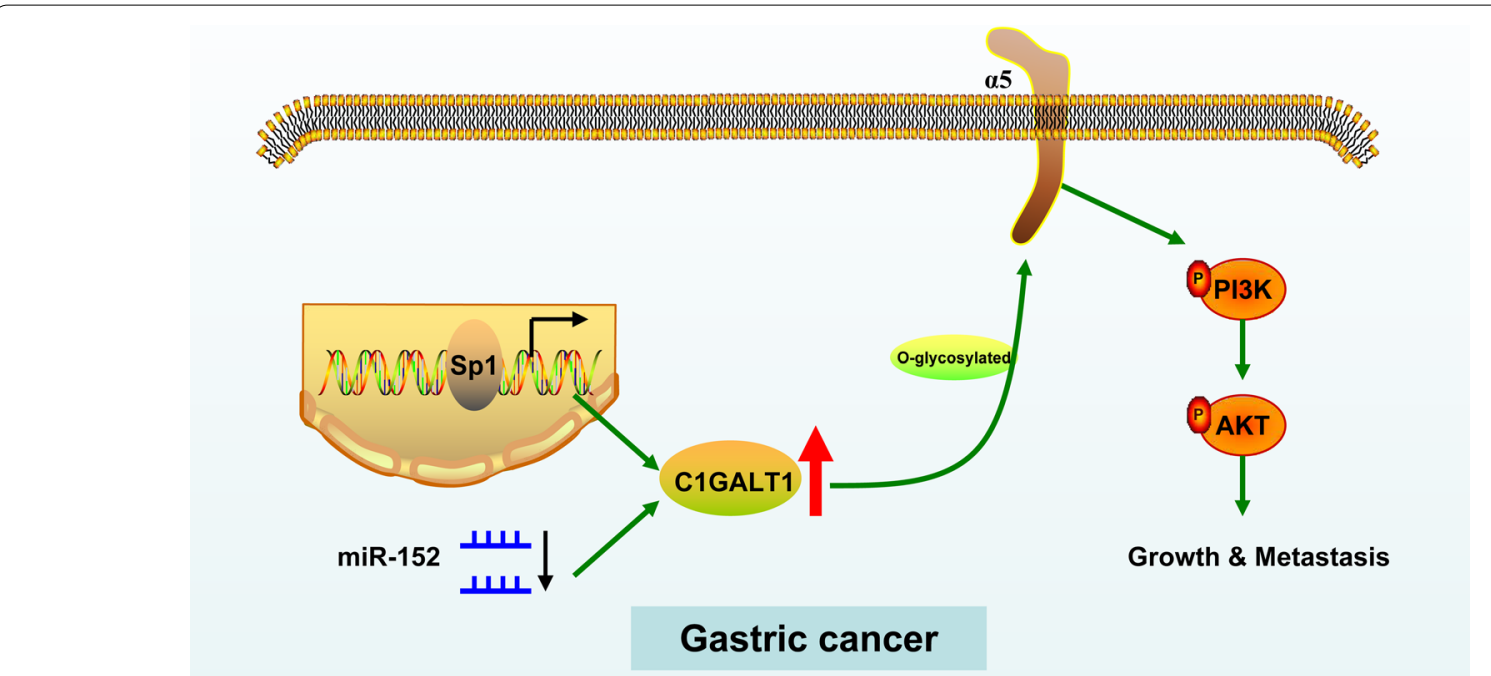

Fig. 7 Diagram of the proposed mechanism showing how C1GALT1 modulates GC progression

miR-152 mimics or NC were co-transfected into cells. Alternatively, C1GALT1 promoter-luciferase reporter plasmid or binding-site mutant plasmid was transfected into cells along with the control plasmid. The luciferase activity was measured at $48 \mathrm{~h}$ after transfection using the Dual-Luciferase Reporter Assay System (Promega).

\section{Animal experiments}

BALB/c nude mice (4-week-old, female) were obtained from the Animal Center of Hubei University of Medicine. Animal experiments were approved by the Animal Health Committee of the Hubei University of Medicine. To assess the tumor growth, $1 \times 10^{7}$ SGC-7901 cells were subcutaneously injected into each mouse. After 4 weeks, mice were killed, and tumors were removed and weighed. To establish the peritoneal metastasis model, $2 \times 10^{6}$ SGC-7901 cells were injected intraperitoneally into each mice. The mice were killed 4 weeks later, and the metastatic nodules were counted.

\section{Statistical analysis}

Data were expressed as the mean \pm SD. Differential gene expression analysis was performed using the MannWhitney U test. The student's t-test or one-way analysis of variance was used for comparison between two groups or more than two groups, respectively. Spearman coefficient was used to analyze correlations. A strong correlation was assumed for $0.7<|\mathrm{r}| \leq 1$, a moderate correlation for $0.5<|\mathrm{r}| \leq 0.7$, a weak correlation for $0.3<|\mathrm{r}| \leq 0.5$, and no correlation for $|\mathrm{r}| \leq 0.3$. Pathologic parameters were analyzed using the $\chi^{2}$ test. The log-rank test was used for Kaplan-Meier survival analysis. A P-value $<0.05$ was considered statistically significant.

\section{Abbreviations}

GC: Gastric cancer; C1GALT1: Core 1 1 1, 3-galactosyltransferase 1; IHC: Immunohistochemistry; QRT-PCR: Quantitative real-time PCR; ChIP: Chromatin immunoprecipitation; PNA: Peanut agglutinin; TCGA: The Cancer Genome Atlas; GTEx: Genotype-Tissue Expression; shRNA: Short hairpin RNA; siRNA: Small interfering RNA; UTR: Untranslated region; CCK-8: Cell Counting Kit-8.

\section{Supplementary Information}

The online version contains supplementary material available at https://doi. org/10.1186/s13578-021-00678-2.

Additional file 1: Figure S1. Scatter diagrams for C1GALT1 expression versus transcription factors expression in GC samples based on the TCGA and GTEx databases. Figure S2. Effect of SP1 on C1GALT1 expression. C1GALT1 mRNA levels were detected by qRT-PCR. siNC, cells transfected with negative control siRNA; si1, cells transfected with SP1 siRNA1; si2, cells transfected with SP1 siRNA2; Ctrl, cells transfected with control plasmid; SP1, cells transfected with SP1 plasmid. ${ }^{*} p<0.05,{ }^{* *} p<0.01$ compared with the siNC or Ctrl group (Student's t-test). Figure S3. Correlation of miR-148b and C1GALT1 expression in GC tissues (Spearman's rank correlation test). Table S1. PNA-binding proteins identified by the proteomic analysis. Table S2. The sequence of shRNAs and siRNAs. Table S3. Primer sequences used in the qRT-PCR analysis.

\section{Acknowledgments}

Not applicable.

\section{Authors' contributions}

$X D$ and $C C$ designed and conducted the experiments; XD, YL, QD, and ZP helped with all experiments; ZL and LS prepared the manuscript. All authors read and approved the final manuscript.

\section{Funding}

This study was supported by the National Natural Science Foundation of China (No. 81902494), the Hubei Provincial Department of Science and Technology Innovation Group program (No. 2019CFA034), the Research Project of 
the Health Commission of Hubei Province (No. WJ2021M048), and the Free Exploration Foundation of the Hubei University of Medicine (No. FDFR201802).

\section{Availability of data and materials}

All data generated during this study are included in this published article and supplementary information files. Further details are available from the corresponding author upon request.

\section{Declarations}

\section{Ethics approval and consent to participate}

Informed consent was obtained from all patients. This study was approved by the Ethics Committee of the Hubei University of Medicine. Animal experiments were approved by the Animal Health Committee of the Hubei University of Medicine.

\section{Consent for publication}

Not applicable.

\section{Competing interests}

The authors declare that they have no competing interests.

\section{Author details}

1 Department of Clinical Oncology, Taihe Hospital, Hubei University of Medicine, Shiyan 442000, Hubei, China. ${ }^{2}$ School of Basic Medical Sciences, Hubei University of Medicine, Shiyan 442000, Hubei, China.

\section{Received: 24 February 2021 Accepted: 15 August 2021}

Published online: 26 August 2021

\section{References}

1. Siegel RL, Miller KD, Jemal A. Cancer statistics, 2020. CA Cancer J Clin. 2020;70:7-30.

2. Wang FH, Shen L, Li J, Zhou ZW, Liang H, Zhang XT,et al. The Chinese Society of Clinical Oncology (CSCO): clinical guidelines for the diagnosis and treatment of gastric cancer. Cancer Commun (Lond). 2019;39:10.

3. Ran A, Guan L, Wang J, Wang Y. GREM2 maintains stem cell-like phenotypes in gastric cancer cells by regulating the JNK signaling pathway. Cell Cycle. 2019;18:2414-31.

4. Yu Z, Li Z, Wang C, Pan T, Chang X, Wang X, et al. Oncostatin M receptor, positively regulated by SP1, promotes gastric cancer growth and metastasis upon treatment with Oncostatin M. Gastric Cancer. 2019:22:955-66.

5. Silsirivanit A. Glycosylation markers in cancer. Adv Clin Chem. 2019;89:189-213.

6. Vajaria BN, Patel PS. Glycosylation: a hallmark of cancer? Glycoconj J. 2017:34:147-56.

7. Dong S, Wang Z, Huang B, Zhang J, Ge Y, Fan Q. Bioinformatics insight into glycosyltransferase gene expression in gastric cancer: POFUT1 is a potential biomarker. Biochem Biophys Res Commun. 2017;483:171-7.

8. Agrawal P, Fontanals-Cirera B, Sokolova E, Jacob S, Vaiana CA, Argibay D, et al. A systems biology approach identifies FUT8 as a driver of melanoma metastasis. Cancer Cell. 2017;31:804-19.

9. Yang Y, Zhang D, Qin H, Liu S, Yan Q. poFUT1 promotes endometrial decidualization by enhancing the $O$-fucosylation of Notch1. EBioMedicine. 2019; 44:563-573.

10. Zilmer M, Edmondson AC, Khetarpal SA, Alesi V, Zaki MS, Rostasy K, et al. Novel congenital disorder of O-linked glycosylation caused by GALNT2 loss of function. Brain. 2020;143:1114-26.

11. Lin MC, Chien PH, Wu HY, Chen ST, Juan HF, Lou PJ, et al. C1GALT1 predicts poor prognosis and is a potential therapeutic target in head and neck cancer. Oncogene. 2018;37:5780-93.

12. Wang Y, Liao X, Ye Q, Huang L. Clinic implication of MUC1 O-glycosylation and C1GALT1 in esophagus squamous cell carcinoma. Sci China Life Sci. 2018;61:1389-95.
13. Chou CH, Huang MJ, Liao YY, Chen CH, Huang MC. C1GALT1 seems to promote in vitro disease progression in ovarian cancer. Int J Gynecol Cancer. 2017;27:863-71.

14. Kuo TC, Wu MH, Yang SH, Chen ST, Hsu TW, Jhuang JY, et al. C1GALT1 high expression is associated with poor survival of patients with pancreatic ductal adenocarcinoma and promotes cell invasiveness through integrin $a(v)$. Oncogene. 2021;40:1242-54.

15. Lee PC, Chen ST, Kuo TC, Lin TC, Lin MC, Huang J, et al. C1GALT1 is associated with poor survival and promotes soluble Ephrin A1-mediated cell migration through activation of EPHA2 in gastric cancer. Oncogene. 2020;39:2724-40.

16. Ju JA, Godet I, Ye IC, Byun J, Jayatilaka H, Lee SJ, et al. Hypoxia selectively enhances integrin $\alpha(5) \beta(1)$ receptor expression in breast cancer to promote metastasis. Mol Cancer Res. 2017;15:723-34.

17. Li XQ, Lu JT, Tan CC, Wang QS, Feng YM. RUNX2 promotes breast cancer bone metastasis by increasing integrin a5-mediated colonization. Cancer Lett. 2016;380:78-86.

18. Pantano F, Croset M, Driouch K, Bednarz-Knoll N, Iuliani M, Ribelli G, et al. Integrin alpha5 in human breast cancer is a mediator of bone metastasis and a therapeutic target for the treatment of osteolytic lesions. Oncogene. 2021;40:1284-99.

19. Wang JF, Wang Y, Zhang SW, Chen YY, Qiu Y, Duan SY, et al. Expression and prognostic analysis of integrins in gastric cancer. J Oncol. 2020; 2020:8862228

20. Cao L, Chen Y, Zhang M, Xu DQ, Liu Y, Liu T, et al. Identification of hub genes and potential molecular mechanisms in gastric cancer by integrated bioinformatics analysis. PeerJ. 2018;6:e5180.

21. Freitas D, Campos D, Gomes J, Pinto F, Macedo JA, Matos R, et al. O-glycans truncation modulates gastric cancer cell signaling and transcription leading to a more aggressive phenotype. EBioMedicine. 2019;40:349-62.

22. Carvalho S, Catarino TA, Dias AM, Kato M, Almeida A, Hessling B, et al. Preventing E-cadherin aberrant N-glycosylation at Asn-554 improves its critical function in gastric cancer. Oncogene. 2016;35:1619-31.

23. Li T, Mo C, Qin X, Li S, Liu Y, Liu Z. Glycoprofiling of early gastric cancer using lectin microarray technology. Clin Lab. 2018;64:153-61.

24. Chugh S, Barkeer S, Rachagani S, Nimmakayala RK, Perumal N, Pothuraju R, et al. Disruption of C1galt1 gene promotes development and metastasis of pancreatic adenocarcinomas in mice. Gastroenterology. 2018;155:1608-24.

25. Wu YM, Liu CH, Huang MJ, Lai HS, Lee PH, Hu RH, et al. C1GALT1 enhances proliferation of hepatocellular carcinoma cells via modulating MET glycosylation and dimerization. Cancer Res. 2013;73:5580-90.

26. Marsico G, Russo L, Quondamatteo F, Pandit A. Glycosylation and integrin regulation in cancer. Trends Cancer. 2018;4:537-52.

27. Hou S, Hang Q, Isaji T, Fukuda T, Gu J. Identification of the minimal $\mathrm{N}$-glycosylation on integrin a5 $\beta 1$ required for its inhibitory effect on EGFR signaling and cell proliferation. Biochem Biophys Res Commun. 2020;523:226-32

28. Hang Q, Isaji T, Hou S, Wang Y, Fukuda T, Gu J. A key regulator of cell adhesion: identification and characterization of important $\mathrm{N}$-Glycosylation sites on integrin a5 for cell migration. Mol Cell Biol. 2017:37:e00558-00516

29. Wilhelm F, Simon E, Böger C, Behrens HM, Krüger S, Röcken C. Novel insights into gastric cancer: methylation of R-spondins and regulation of LGR5 by SP1. Mol Cancer Res. 2017;15:776-85.

30. Huang M, Hou J, Wang Y, Xie M, Wei C, Nie F, et al. Long Noncoding RNA LINC00673 is activated by SP1 and exerts oncogenic properties by interacting with LSD1 and EZH2 in gastric cancer. Mol Ther. 2017:25:1014-26.

31. Feng F, Liu H, Chen A, Xia Q, Zhao Y, Jin X, et al. miR-148-3p and miR$152-3 p$ synergistically regulate prostate cancer progression via repressing KLF4. J Cell Biochem. 2019;120:17228-39.

32. Sun J, Tian $X$, Zhang J, Huang $Y$, Lin $X$, Chen L, et al. Regulation of human glioma cell apoptosis and invasion by miR-152-3p through targeting DNMT1 and regulating NF2: MiR-152-3p regulate glioma cell apoptosis and invasion. J Exp Clin Cancer Res. 2017;36:100.

33. Ma P, Li L, Liu F, Zhao Q. HNF1A-induced IncRNA HCG18 facilitates gastric cancer progression by upregulating DNAJB12 via miR-152-3p. Onco Targets Ther. 2020;13:7641-52. 
34. Shen L, Xia M, Deng X, Ke Q, Zhang C, Peng F, et al. A lectin-based glycomic approach identifies FUT8 as a driver of radioresistance in oesophageal squamous cell carcinoma. Cell Oncol (Dordr). 2020:43:695-707.

35. Dong X, Luo Z, Liu T, Chai J, Ke Q, Shen L. Identification of integrin beta1 as a novel PAG1-interacting protein involved in the inherent radioresistance of human laryngeal carcinoma. J Cancer. 2018;9:4128-38.
36. Chen K, Zhu L, Guo L, Pan YB, Feng DF. Maf1 regulates dendritic morphogenesis and influences learning and memory. Cell Death Dis. 2020;11:606

\section{Publisher's Note}

Springer Nature remains neutral with regard to jurisdictional claims in published maps and institutional affiliations.
Ready to submit your research? Choose BMC and benefit from:

- fast, convenient online submission

- thorough peer review by experienced researchers in your field

- rapid publication on acceptance

- support for research data, including large and complex data types

- gold Open Access which fosters wider collaboration and increased citations

- maximum visibility for your research: over $100 \mathrm{M}$ website views per year

At BMC, research is always in progress.

Learn more biomedcentral.com/submissions 